\title{
El Sistema de Acreditación de Carreras de Grado del MERCOSUR (ARCU-SUR) en la Facultad de Agronomía de la Universidad de la República - Uruguay
}

\author{
Ernesto Domínguez Misa
}

Resumen: La participación en el ARCU-SUR, plantea en las carreras el debate sobre la calidad y su aseguramiento, así como la respuesta institucional a la misma. Irrumpen en la agenda temas derivados como el reconocimiento de títulos, la nueva institucionalidad, la relación público - privado y universidad - estado. Este artículo realizará una revisión sobre los fundamentos, resultados e impactos producidos en la Facultad de Agronomía de la Universidad de la República, Uruguay, por el Mecanismo Experimental de Acreditación de Carreras de Grado del MERCOSUR (MEXA) y el Sistema de Acreditación ARCU-SUR, centrándose en los efectos derivados, pero explora retrospectivamente la evolución institucional en las políticas de evaluación .

Palabras clave: Acreditación, Evaluación. ARCU-SUR.

\section{O Sistema de Acreditação de Cursos de Graduação do MERCOSUL (ARCU-SUL) na Faculdade de Agronomía da Universidade da República - Uruguai}

Resumo A participação no ARCU-SUR coloca nos cursos superiores o debate sobre a qualidade e garantia, bem como a resposta institucional a ela. Entram as questões suscitadas quanto ao reconhecimento dos diplomas, as novas instituições, a agenda de relação público - privada e universidade - estado. Este artigo fará uma revisão dos fundamentos, resultados e impactos na Faculdade de Agronomia da Universidade da República, Uruguai, pelo Mecanismo Experimental de Acreditação de Cursos de Graduação do MERCOSUL (MEXA) e o Sistema de Acreditação ARCU-SUR e concentrando-se nos efeitos colaterais, mas em retrospecto explora a evolução institucional nas políticas de avaliação.

Palavras-chave: Acreditação. Avaliação. ARCU-SUR.

\section{MERCOSUR Degree Accreditation System (ARCU-SUR)}

\section{at the Faculty of Agronomy of the University of the Republic - Uruguay}

Abstract: Participation in the ARCU-SUR poses in racing the debate on the quality and assurance as well as the institutional response to it. Burst into the issues arising as the recognition of diplomas, the new institutions, the public-private and university-state relations. This article presents a review of the fundaments, results and impacts at the Faculty of Agronomy of the University of the Republic, Uruguay, by the Experimental Mechanism of Accreditation of Degree of MERCOSUR (MEXA) Accreditation System ARCU -SUR and focusing on spillover effects, but in retrospect explores the institutional evolution in policy evaluation.

Key words: Accreditation. Evaluation. ARCU-SUR.

Este é um artigo publicado em acesso aberto sob uma licença Creative Commons https://creativecommons.org/licenses/by-nc/4.0/ 


\begin{abstract}
Por mais fraca que seja a influência que a minha voz possa ter nas atividades públicas, pretende ser uma contribuição às reformas que hão de vir. Pois, manda a prudência que antes de reformar se avalie, se prepare o retrato falado das instituições de tal sorte que sobre elas todos tenham conhecimiento. E que, ao producir conhecimento sobre a universidade, todos possam, no exercício pedagógico das regras do jogo democrático, ser participantes ativos de sua transformação (LEITE, 2005).
\end{abstract}

\title{
1 Introdução
}

Este artículo realizará una revisión y reflexión sobre los fundamentos, resultados e impactos producidos en la Facultad de Agronomía de la Universidad de la República (UdelaR), por su participación en el Mecanismo Experimental de Acreditación de Carreras de Grado del MERCOSUR (MEXA) y el Sistema de Acreditación (ARCU-SUR). Se centra en las acciones de mejora producidas, la articulación con el Programa de Evaluación Institucional de la propia Universidad (PEI), y en la construcción de una cultura de la evaluación.

Se toma como territorio de análisis la carrera de Agronomía de la UdelaR y se centra en los efectos derivados, explorando retrospectivamente la evolución institucional en las políticas de evaluación. Se ponen de relevancia los cambios generados en la Facultad y la carrera, y el aporte a una cultura de la evaluación y la calidad, así como la repercusión en políticas de impacto nacional y regional.

La participación en el Sistema de Acreditación del MERCOSUR (ARCU-SUR), plantea el debate sobre la calidad de las carreras y los mecanismos de aseguramiento de la misma, así como la respuesta institucional al mejoramiento de la enseñanza. Irrumpen en la agenda universitaria temas derivados de la acreditación, como el reconocimiento de títulos, la nueva institucionalidad, la relación público - privado y universidad - estado.

Se estudia esta titulación, por ser la primera que se convocó a la acreditación en el MERCOSUR, en la que inicialmente se realizó un pre-test de validación, el MEXA y finalmente el ARCU-SUR. Por otro lado, es una Facultad que participó activamente del Programa de Evaluación Institucional de la UdelaR y cumplió todas las etapas previstas del mismo. Finalmente, las posiciones institucionales frente a la acreditación y la evaluación han sido proactivas y de apoyo. Lo anterior posiciona a la Facultad como una de las de mayor experiencia, reflexión y aporte a la temática en la Universidad.

El tema de la calidad, que atraviesa todo este trabajo, será considerado de acuerdo a lo planteado por Dias Sobrinho (1995), en tanto construcción social, que varía según los intereses de los grupos de adentro y de afuera de la institución, que refleja las características de la sociedad que se desea para hoy y se proyecta hacia el futuro. No es 
un concepto unívoco y fijo sino que debe ser construido a través de consensos y negociaciones entre los actores. A partir de esto, la UdelaR se ha plegado a la definición establecida por la Unesco (1998), para la que "la calidad de la enseñanza superior es un concepto pluridimensional que debería comprender todas sus funciones y actividades: enseñanza y programas académicos, investigación y becas, personal, estudiantes, edificios, instalaciones, equipamiento y servicios a la comunidad y al mundo universitario”.

\section{Presentación de la carrera de Agronomía de la UdelaR}

La carrera pública de Ingeniero Agrónomo de la Universidad de la República es la única que existe en Uruguay y está instalada en Montevideo, con cuatro sedes en el interior del país. El tiempo de duración de la carrera en su plan de estudios es de 5 años, con 4000 horas en total. El perfil del ingeniero agrónomo lo define como el profesional universitario preparado para comprender, manejar, mejorar, y transformar sistemas de producción agropecuarios con el objeto de servir al bienestar social y al desarrollo nacional sostenido (FACULTAD DE AGRONOMÍA, 2009b).

Se funda la Facultad de Agronomía a partir del Decreto Presidencial del 15 de setiembre de 1906 durante la presidencia de José Batlle y Ordoñez y el rectorado del Dr. Eduardo Acevedo, estando a cargo de la dirección de la Facultad el Dr. Alejandro Backhaus y las clases se inician en marzo de 1907. Las tareas iniciales serán: formar agrónomos, provocar investigaciones científicas originales y estimular el desarrollo de la agronomía nacional. Se preveían tres tipos de egresado, aunque la prioridad era para los ingenieros agrónomos que debían completar cuatro años de cursos y un quinto año destinado a elaborar y defender su trabajo de tesis. En agosto de 1907 se funda la Asociación de Estudiantes de Agronomía, que continuará hasta nuestros días como la organización representativa de los estudiantes de la carrera.

Por Ley del 30 de setiembre de 1911 se crean las estaciones agronómicas, estableciéndose en 1914 La Estanzuela, en Colonia. En 1925 la Escuela de Agronomía pasa a ser Facultad y en 1961 la institución pasa a llamarse Centro de Investigaciones Agrícolas Alberto Boerger (CIAAB) y amplía sus cometidos a los rubros ganaderos con énfasis en su base forrajera (BRASESCO, 2007).

Existen hoy dos carreras de ingeniería, dos licenciaturas de grado y dos tecnólogos. A nivel de posgrado se dicta un doctorado, tres maestrías, dos diplomas de especialización y a su vez en forma compartida con otras facultades integra una maestría y un diploma de 
El Sistema de Acreditación de Carreras de Grado del MERCOSUR (ARCU-SUR) en la Facultad de Agronomía de la Universidad de la República - Uruguay

especialización. Asimismo se realizan importantes actividades de investigación y extensión universitaria de las que se nutre la formación impartida.

En el año 2012 la Facultad contaba con aproximadamente 2300 estudiantes de grado, se produjeron 154 egresos y en el año 2013 ingresaron 482 estudiantes. El cuerpo docente en el año 2013 se compone de 375 cargos y 11674 horas con 71 profesores de dedicación total, y asimismo el cuerpo no docente se compone de 291 funcionarios. Respecto a la infraestructura edilicia la Facultad cuenta con un edificio central y una granja en Montevideo, tres estaciones experimentales en Salto, Paysandú y Cerro Largo y un Centro Regional en Canelones, que totalizan $32862 \mathrm{~m}^{2}$ (Universidad de la República, 2015).

\section{Fundamentos de la participación de la carrera de Agronomía en la acreditación regional}

En el proceso de integración de la Facultad de Agronomía en la acreditación regional, fue importante que la misma se encontraba integrada al Foro Regional de Facultades de Agronomía del MERCOSUR, creado casi desde la firma del Tratado de Asunción, y que tenía como objetivos la integración regional de las carreras de Agronomía. En dicho Foro se empezó a discutir, incluso anteriormente a la creación del MEXA, el reconocimiento de los títulos en la región como un objetivo político, por lo que se allanaba el posicionamiento frente a las iniciativas del SEM (ABADIE, 2009).

Un segundo factor para integrarse fue el reconocimiento de la efectiva movilidad profesional a partir de la creación del MERCOSUR, que estimulaba la necesidad de garantizar la calidad de esos profesionales que ya circulaban por la región. Este fenómeno estaba en la preocupación del Foro de Facultades, lo que llevó a impulsar políticas de evaluación para encontrar mecanismos de reconocimiento de los títulos.

El tercer motivo, era el mejoramiento de la calidad de la carrera en sí misma, a partir de la conjunción de muchos de los otros objetivos como la integración regional, la cooperación académica, la sistematización de criterios de calidad, entre otros.

Como cuarto motivo encontramos la validación política tanto interna como pública en tanto rendición de cuentas a la sociedad. Esto está inserto en una política universitaria más general, que buscaba anticiparse a la presión social y fortalecer la legitimidad de la institución. 
El quinto, y último factor o motivo de impulso de las políticas de evaluación y acreditación, se vincula al convencimiento de cómo se construye una agenda política y de acción universitaria en forma participativa.

\section{EL MEXA en la carrera de Agronomía}

La carrera de Agronomía presentaba antecedentes en procesos de evaluación, que se remontan a la década de los 90 donde por un lado se desarrollan actividades de evaluación del plan de estudio, y por otro en el año 1998 la UdelaR pone en funcionamiento el Programa de Evaluación Institucional, al que la Facultad se incorpora voluntariamente entre 1999 y el año 2000, cumpliendo con las etapas de autoevaluación y evaluación externa previstas por el Programa.

Cuando se comienza a desarrollar el sistema regional de acreditación, la titulación de Agronomía es de las primeras propuestas por los países para ser incorporada al mismo por su vínculo productivo tanto nacional como regional. Al ser en el Uruguay la única carrera de ingeniería agronómica, su participación se transformó en estratégica para el país.

Claramente los tres procesos son diferentes, pero tienen un conjunto de actividades y necesidades de información común. Este hecho permitió que los procesos se retroalimentaran. Por un lado lo enriquecieron al incorporar la dimensión regional, ya que al tener que cumplir un cronograma de actividades acordado en el ámbito regional marcó un ritmo que debió seguirse. Las actividades de evaluación del plan de estudio, y propiamente las relacionadas con la autoevaluación, agudizaron un aspecto central del proceso que ha sido la autoevaluación y la búsqueda de las oportunidades de mejora, lo que contrarrestó posibles actitudes autocomplacientes (FACULTAD DE AGRONOMÍA, 2001b, p. 9).

En octubre del año 2003 la Facultad inscribe a la carrera en el proceso piloto de Acreditación de Carreras del MERCOSUR (MEXA), y se trabaja desde el decanato actualizando el Informe Institucional 2001, documento que la facultad realizó para el PEI, a los efectos de adecuarlo al formato solicitado.

Se encuentran diversas manifestaciones de la implicancia de las autoridades y en general de los docentes, aunque no se pudo constatar el mismo entusiasmo a nivel estudiantil. El 29 de diciembre de 2003 se instala la nueva Asamblea del Claustro de la Facultad y este órgano asume la responsabilidad de conducir el proceso de acreditación. 
El Sistema de Acreditación de Carreras de Grado del MERCOSUR (ARCU-SUR) en la Facultad de Agronomía de la Universidad de la República - Uruguay

Finalmente el 2 de febrero de 2004, el Claustro aprueba los documentos definitivos para la acreditación y el 6 de febrero lo hace el Consejo de Facultad. Teniendo en cuenta lo establecido por el protocolo, el 9 de febrero de 2004 se firma el acuerdo entre la Facultad y la Comisión que regula el proceso a seguir y el 12 de febrero se entregan los documentos originales. El 29, 30 y 31 de marzo 2004 se produce la visita de los pares evaluadores y el 14 de junio de 2004 se emite el dictamen y la resolución favorable por la Comisión Ad Hoc. Dicha resolución acreditaba a la carrera de Agronomía de la UdelaR por el plazo de cinco años y le solicitaba que en un plazo de dos años se presentara un informe acerca de la forma en que ha abordado las recomendaciones realizadas en dicha resolución (COMISIÓN AD HOC, 2004).

\section{El ARCU-SUR en la carrera de Agronomía}

En agosto del 2008 se realiza en todos los estados parte y asociados del MERCOSUR la convocatoria a carreras de Agronomía al proceso de acreditación en el Sistema ARCU-SUR. El 18 de agosto de 2008 el Consejo de la Facultad de Agronomía manifiesta la voluntad de participar en dicha convocatoria.

La Facultad encarga la coordinación de la acreditación a la Comisión de Evaluación y Acreditación de carácter cogobernado y nombra como coordinador del proceso al director de la Unidad de Enseñanza. Como apoyo operativo se constituye un equipo de trabajo y se asignan recursos materiales y económicos.

Una vez realizado el proceso de autoevaluación, el Consejo aprueba en mayo del 2009, el estudio FODA y el informe de autoevaluación a presentar. La visita de pares externos se realiza entre el 7 y el 10 de setiembre de ese año. Por último el 9 de noviembre la Comisión Ad Hoc emite el acuerdo de acreditación n²/09 donde acredita por 6 años a la carrera de Agronomía de la UdelaR (COMISIÓN AD HOC, 2009).

En el año 2013 se comienzan las actividades preparatorias para la presentación a la segunda acreditación ARCU-SUR que se convocó en setiembre del 2015. Coherente con la experiencia acumulada y la cultura institucional construida, en abril del 2013 se aprueba un cronograma de trabajo, y en agosto del 2014 el Consejo de la Facultad expresa su voluntad de participar en dicha convocatoria. 


\section{Componentes de la autoevaluación}

La evaluación puede tener dos objetivos, por un lado valorar la eficiencia y eficacia de la carrera y por otro la mejora institucional. Los procesos en que participó la carrera de Agronomía fueron encarados por la misma priorizando la mejora, más allá de los objetivos propios de los sistemas de acreditación.

\subsection{El modelo}

Al participar tanto del PEI, como el MEXA y el ARCU-SUR, la institución recibió los lineamientos y pautas metodológicas ya definidas. En el primero con criterios más abiertos y en los segundos con un carácter más cerrado, aunque en ambos casos incorporaban indicadores cuantitativos y cualitativos. A pesar de las diferencias, la Facultad le aplicó su propio enfoque, maximizando los esfuerzos, potenciando las asimetrías y priorizando la secuencia de experiencias como una continuidad del aseguramiento de la calidad y la mejora.

\subsection{El informe}

Si bien en los tres casos en que se construyó un informe de autoevaluación, presentaron singularidades, en todos ellos cumplieron con las características principales de este tipo de documento. Se presentaron las actividades, organización y funcionamiento de la carrera en forma cuantitativa y cualitativa realizando un análisis y apreciación crítica sobre la realidad incorporando sugerencias de mejora como resultados de la reflexión.

Los tres informes tienen una impronta estilística distinta, siendo el del MEXA el más diferenciado, ya que se optó por redactarlo a partir de las sugerencias de mejora realizadas en el PEI (FACULTAD DE AGRONOMÍA, 2004). Esta diferencia también expresó desviaciones distintas, siendo los dos primeros informes más críticos, mientras que el último realiza una presentación mayormente positiva. Indudablemente los informes permiten conocer más profunda e intensamente a la carrera, en sus problemas, explicación de los mismos y posibles proyecciones a futuro.

\subsection{El equipo técnico}

La institución optó por utilizar recursos humanos de la propia carrera, luego de una fallida experiencia de contratar especialistas externos. Se organizó una estructura con un 
El Sistema de Acreditación de Carreras de Grado del MERCOSUR (ARCU-SUR) en la Facultad de Agronomía de la Universidad de la República - Uruguay

coordinador técnico del proceso, que en todos los casos era con formación en el tema, dando cuenta de que la solidez del informe necesita de recursos humanos con saber en la temática. Esta solución logró darle operatividad al proceso, coherencia interna al informe y consistencia técnica.

\subsection{Construcción de la información}

La institución, y los principales operadores de la autoevaluación, demuestran conciencia de la importancia de la información para lograr los objetivos. En tal sentido, los informes de la carrera de Agronomía presentan una importante dosis de información cuantitativa. Conseguir esta información, para tratar de escapar al subjetivismo, implicó un desafío, debido a la falta de datos y sistematización de la información existente. Marisquirena (2011) llama la atención sobre la dificultad para reunir la información interna por motivos de dispersión, doble registración, inconsistencias y falta de sistematización, y la información externa por diversidad de actores.

\subsection{Compromiso de las autoridades}

La participación de las autoridades en los procesos, fue de alto compromiso y con una gran implicancia. El Decano asumió no solo una actitud participativa sino de liderazgo, y el Consejo realizó un seguimiento directo de las actividades de evaluación, tomando las resoluciones necesarias, asimismo el Claustro destinó tiempo y recursos en la consideración de los temas que necesitaban de su participación. Así fue registrado por los pares externos en su informe (COMISIÓN AD HOC - pares externos, 2009).

\subsection{Participación de los actores}

La validación de la participación se realiza a partir de cuál y cómo sea esa participación y no en una relación cuantitativa. Desde este punto de vista, la institución ha utilizado diversidad de instrumentos, algunos de participación directa como reuniones, asambleas, entrevistas, etc., otros indirectos como encuestas y relevamientos. Los actores tienen una percepción positiva del empoderamiento que hizo la comunidad del proceso. Los estudiantes participaron limitadamente, no realizaron oposición al mismo aunque no era su principal objetivo. La directiva y la estructura militante de la Asociación de Estudiantes de Agronomía (AEA) estaba comprometida con llevar adelante el proceso a pesar de ser más exigente en cuanto a la visión de lo logrado. 


\section{Síntesis de los resultados del proceso de acreditación}

Se entenderá resultados en la conceptualización que hacen las políticas públicas en tanto materializaciones físicas o realizaciones provocadas por una política. Como en toda historia institucional, en muchos casos los mismos fueron reflejo directo del proceso de acreditación, en otros lo que hicieron dichos procesos fue catalizar iniciativas en discusión, y por último en algunos casos sirvieron de impulso de transformaciones que no contaban con el apoyo consensual de la comunidad académica.

Dimensión institucional. Uno de los resultados más importantes por su efecto y por su potencial de corto y mediano plazo, fue la incorporación de la planificación como una herramienta de gestión académica y administrativa, con una expresión que fue un Plan Estratégico. Durante el proceso de autoevaluación, se detectó que la carrera tenía la definición de misión en su plan de estudios pero no contaba con un Plan Estratégico ni estaba previsto ningún mecanismo para la generación del mismo (FACULTAD DE AGRONOMÍA, 2004).

La planificación se instaura como una herramienta permanente y en diversos niveles de la organización, desde lo macro hasta las unidades académicas. Esta política es reconocida como una ganancia derivada de la evaluación, que permite dar insumos para la administración y también como apoyo a la actividad del cogobierno.

La Facultad desde los primeros informes de autoevaluación, detectó inconvenientes en la organización y gestión de la carrera, para lo que se contrató una consultoría que recomendó medidas sobre la gestión institucional en la que se ha presentado al Consejo el informe Políticas Generales de la Facultad sobre Gestión y se aprobó un organigrama del área administrativa de Montevideo.

A pesar de las medidas tomadas, el acuerdo de acreditación 2009 vuelve a "señalar como una oportunidad de mejora fortalecer los mecanismos de evaluación de la gestión administrativa y académica" (COMISIÓN AD HOC, 2009).

Dimensión proyecto académico. Esta es una dimensión ampliamente considerada en la Facultad, tanto en la discusión del plan de estudio, de programas y de que y como se enseña. Esto puede justificar que la percepción de los actores sea que no hubo demasiados efectos en los contenidos de la carrera de Agronomía ni en su estructura académica a partir de la acreditación. 
El Sistema de Acreditación de Carreras de Grado del MERCOSUR (ARCU-SUR) en la Facultad de Agronomía de la Universidad de la República - Uruguay

Las observaciones de la acreditación ARCU-SUR en el proyecto académico no cuestionaron el plan de estudios sino que remiten más a cuestiones de organización académica o de prácticas docentes. De todas formas, la Facultad se encuentra inserta en debates sobre el cambio del plan de la carrera desde hace varios años, y a pesar de los ajustes parciales que se han realizado, no se ha logrado al día de hoy reformar el mismo.

Otro resultado derivado de la reflexión sobre el perfil del egresado fue el planteo de una política de diversificación de la oferta académica de la enseñanza de grado. En el año 2010 se completa la carrera de Ingeniero Agrónomo en la sede Noroeste, se produce la apertura de la Licenciatura en Diseño de Paisaje, la Licenciatura en Viticultura y Enología y la Licenciatura en Gestión Ambiental. También se promovieron titulaciones terciarias técnicas y se abren las carreras de Tecnólogo Agroenergético (2009), Tecnólogo Cárnico (2010) y Tecnólogo en Madera (2011).

El informe de evaluación de pares del ARCU-SUR marcaba problemas en la carga horaria de los estudiantes, y esto fue atendido por el Consejo realizando una reforma de las horas de los cursos. Respecto a la duración de la carrera, ya en el informe de autoevaluación del 2001 fue detectado como un problema y se recomendaba tomar medidas para que la duración nominal y real de la carrera tendieran a coincidir.

Ante la sugerencia de fortalecer la ética en los procesos de enseñanza, se incorpora la temática en diversos cursos de la carrera.

Respecto a la relación con la investigación, en la autoevaluación 2004, se planteó que entre las recomendaciones de la autoevaluación 2001 se destacaba "estimular el fortalecimiento, a través de contactos realizados bajo marcos formales, de la articulación interinstitucional que asegure a la Facultad la adecuada identificación de demandas de tecnologías" (FACULTAD DE AGRONOMÍA, 2001b, p. 9), y teniendo en cuenta la evaluación externa, la misma destaca la baja pertinencia de la investigación en relación a los problemas de interés nacional; la carencia de un proceso de planificación y priorización formal, que atenta contra la relevancia y el uso de los recursos; y la escasa coordinación con otros servicios de la Universidad o con instituciones externas.

Para enfrentar estas observaciones realizadas, la Facultad realizó un esfuerzo importante en dos ejes: $1^{\circ}$ convocar desde el Área Agraria, y en el marco de la Comisión Social Consultiva de la UDELAR, la formación de Mesas por cadena agropecuaria; y $2^{\circ}$ la formación de los grupos interdisciplinarios de investigadores vinculados a cada cadena.

En el informe de autoevaluación 2009 se presenta un enfoque distinto de las actividades de investigación respecto a lo indicado en el 2004, y en dicho informe luego de 
una descripción de las importantes iniciativas y proyectos, se concluye que existió una fuerte mejora de la investigación y su vínculo con el aparato productivo y social (FACULTAD DE AGRONOMÍA, 2009a).

Respecto a la función de la extensión, así como su articulación con la enseñanza, en ninguno de los dictámenes de acreditación es mencionada como una debilidad ni se realizan sugerencias de mejora. La preocupación por la extensión es de larga data en la Facultad de Agronomía, y es en uno de los temas que se puede considerar que los procesos de evaluación y acreditación ayudaron a catalizar un impulso que ya existía.

Dimensión comunidad académica. Algunos de los efectos producidos por la acreditación generaron protocolos e instrumentos que se incorporaron en la gestión de la carrera, por lo que tendrán un efecto de carácter permanente. En esta línea se organizaron dos sistemas de manejo de datos e información, uno dirigido a docentes, el sistema de información académica; y el otro a los egresados, el sistema de seguimiento.

Complementando el manejo de la información docente, y a raíz del requisito de los indicadores ARCU-SUR, la carrera organiza y uniformiza la disponibilidad de todos los curriculum de los docentes.

El seguimiento de egresados, fue sugerido en el considerando III, literal d. de la resolución de acreditación del 14 de junio de 2004, a partir de lo manifestado por el comité de pares, y como respuesta se crea la Unidad de Difusión y Comunicación en el año 2009.

Una tercera demanda de los pares reflejada en el dictamen, fue desarrollar un sistema dinámico de calificación docente, para lo que el Consejo, el 21 de agosto de 2006 aprueba pautas que definen las actividades académicas realizadas por los docentes tanto para el ingreso como para la evaluación durante las reelecciones. Complementariamente se aprueba el 14 de mayo de 2007 una planilla que otorga puntaje relativo y coeficientes de corrección por grado para ser aplicada de acuerdo a las actividades docentes de enseñanza, investigación, extensión y cogobierno.

El dictamen de la Comisión Ad Hoc de 2004 sugería que "dirija la formación superior de los docentes a áreas de vacancia o de innovación en los campos ocupacionales de la agronomía", para lo que la Facultad crea un Grupo de Trabajo Interdepartamental para desarrollar actividades en el área de calidad en los procesos agronómicos, integrándose el tema en diversos cursos. 
El Sistema de Acreditación de Carreras de Grado del MERCOSUR (ARCU-SUR) en la Facultad de Agronomía de la Universidad de la República - Uruguay

La formación pedagógica es considerada desde la autoevaluación del PEI en 2001, en respuesta a esta temática existía el Proyecto de Formación Didáctico Pedagógica del Área Agraria, el Consejo de la Facultad resolvió, el 29 setiembre y el 13 de octubre de 1997, la obligatoriedad para sus docentes de tomar cursos didáctico-pedagógicos.

Por otra parte, el fenómeno de la movilidad que no se encuentra demasiado difundido en la región, es paulatinamente incorporado en las políticas de las instituciones universitarias. El SEM organizó como otra de sus líneas estratégicas, un programa de movilidad vinculado a las carreras acreditadas. La participación en el MEXA y el ARCU-SUR permitió a la carrera de Agronomía su integración al Programa de Movilidad MARCA, dirigido a estudiantes y docentes de carreras acreditadas en el MERCOSUR.

\section{Dimensión infraestructura física y logística. En esta dimensión la institución} reconoce el mayor efecto del proceso de evaluación institucional y de acreditación. Si bien la Facultad era consciente de debilidades en la infraestructura, en particular en la disponibilidad de espacios especializados para la enseñanza, la autoevaluación sirvió para que lo racionalizara y explicitara al punto que aunque el informe de los pares es muy positivo, la carrera en su respuesta al mismo, se compromete a trabajar, entre otros, en la mejora de la infraestructura edilicia y el equipamiento para las funciones de enseñanza, investigación y extensión.

El informe de pares del MEXA, sugirió la necesidad de cambios en la biblioteca para adecuarla a los requerimientos universitarios, lo que fue reafirmado en el dictamen de acreditación 2004. Al momento de la visita de pares estaba iniciándose la aplicación del Proyecto de Mejora de la Biblioteca, con importantes avances en equipamiento, área recursos humanos y acervo.

En los aspectos edilicios, los sucesivos dictámenes de acreditación marcan recurrentemente sugerencias de mejora. Desde antes de la primer visita de pares se estaba ejecutando un plan de mejora de utilización de los espacios disponibles, elaborado a partir de una consultoría externa. A partir del mismo se aumentó el número de aulas, se acondicionaron una serie de espacios interiores y exteriores, se sustituyó el equipamiento mobiliario y se realizaron importantes obras de adecuación y ampliación de locales especializados.

\section{Los objetivos MEXA y ARCU-SUR en la carrera de Agronomía}




\subsection{Instalación de la cultura de la evaluación}

La acumulación de experiencias y actividades han logrado avanzar en la instalación de la cultura de la evaluación en la institución hasta el punto que la realización de autoevaluación ya es considerado parte de las actividades normales. La participación y los efectos positivos del proceso de acreditación regional, es un tema aceptado y consensuado tanto en la Facultad de Agronomía como en general en la UdelaR.

El desarrollo de esta línea de trabajo llevó a formalizar la estructura de la Unidad de Planeamiento y Evaluación Institucional, como forma de organizar y articular la planificación. Otro de los elementos, es el plan anual de trabajo de cada docente, que se organizó en el Sistema de Gestión Académica en forma informatizada que permite además de evaluar a los docentes, planificar las actividades de las distintas unidades académicas.

\subsection{Mejora de la calidad}

El PEI, el MEXA y el ARCU-SUR dejaron carreras e instituciones que transitaron una experiencia novedosa, con planes de mejora formulados a los efectos de la superación de la calidad en la educación.

La Facultad de Agronomía produjo una serie de resultados, a partir de iniciativas y cambios vinculados a los procesos de acreditación, que tuvieron como objetivo principal la mejora de la calidad de la carrera. Se pueden identificar impactos con permanencia, entre los principales destacaremos dos, por una lado la política de posgrados, en específico los programas de maestría y doctorado con la consecuente repercusión en la cantidad de docentes formados, así como la investigación producida. Por otro lado, la mejora de la biblioteca, los espacios físicos y la logística.

\subsection{Aporte al proceso de integración}

Este objetivo establecido en el Acuerdo de Entendimiento del ARCU-SUR (RME, 2008) refiere a aspiraciones de mayor alcance que exclusivamente lo educativo, pero indudablemente a través de la educación se fortalece la integración regional. La actividad académica establecida a partir del proceso de acreditación genera aprendizajes, cooperación, redes y vínculos importantes. El desarrollo del ARCU-SUR ha logrado construir un relacionamiento a nivel institucional y personal entre los principales 
El Sistema de Acreditación de Carreras de Grado del MERCOSUR (ARCU-SUR) en la Facultad de Agronomía de la Universidad de la República - Uruguay

referentes en la temática. Varios sectores del MERCOSUR, ponderan el grado de avance que ha logrado el SEM, y dentro de éste en especial el área de educación superior.

\subsection{Apoyo a mecanismos de reconocimiento de títulos universitarios en la región}

El objetivo del Sistema ARCU-SUR de apoyo a los mecanismos de reconocimiento de títulos universitarios en la región es, junto con la mejora de la calidad, fundamental en la intencionalidad política de los países de la región de lograr la movilidad de profesionales en el MERCOSUR.

La Facultad de Agronomía se posiciona en un total apoyo a aprobar el reconocimiento de los títulos de las carreras acreditadas. Se reconoce una realidad que ya está pasando dentro del ejercicio profesional.

\subsection{Fortalecer competencias técnicas}

Como consecuencia de los diversos procesos de evaluación y acreditación, existe una red inorgánica de académicos y gestores insertos en diversos servicios de la institución que presenta formación y disposición a trabajar activamente en la construcción de este enfoque de la educación superior.

La experiencia del PEI, inició un núcleo de académicos, con conocimiento y experiencia en la temática, y esta formación de recursos humanos se ha visto fortalecida a partir de la participación regional, que dejó “como virtud del proceso, la importante ampliación de la masa crítica de personas capacitadas para encarar rigurosamente los procesos de aseguramiento de la calidad de la educación universitaria. Esa disponibilidad de recursos, que ya hoy es cualitativamente superior a la existente hace apenas unos años, podrá servir al imprescindible delineamiento de políticas de mejoramiento de la educación superior en el país” (LANDINELLI, 2007, p. 31).

\section{Consideraciones finales}

La dimensión política. Las primeras consideraciones se vinculan a las concepciones políticas y filosóficas que la UdelaR puso en juego en estos procesos. Profundizado el comportamiento de la misma y de la Facultad de Agronomía, quedó expuesto que se concibe a la educación como un bien público y un derecho de la sociedad. La institución enfocó los procesos de acreditación en forma consecuente con este posicionamiento ideológico. 
La defensa de estas ideas mantuvo latente una tensión con un sistema como el ARCUSUR, la que se manejó a partir de un equilibrado acuerdo de todos los actores en dicho sistema. Por lo tanto lo que Boaventura de Sousa Santos (2010) define como crisis institucional, se resuelve en el vínculo ARCU-SUR - Universidad a partir del reforzamiento de la autonomía de ésta última, la construcción del sistema interinstitucionalmente y particularmente por la implicancia de la institución para definir el ARCU-SUR como un verdadero sistema de mejoramiento de la calidad.

La UdelaR, no sin contradicciones, ha intentado manejar las exigencias de la sociedad de mayor rendición de cuentas, de mejora de la calidad y del derecho a conocer dicha calidad, a través de iniciativas como el Programa de Evaluación Institucional, el ingreso a la acreditación regional y los proyectos de reforma universitaria. Estas iniciativas afirman lo planteado por Leite (2005), sobre la necesidad de que la universidad debe autorrevolucionarse como forma de reafirmación de su autonomía y valor social. Los mayores avances y decididos apoyos se han encontrado en las Facultades como unidades autónomas, en tanto debe prevenirse que la respuesta central no ha tenido la energía ni el compromiso necesario.

Estando la calidad en el centro de los sistemas de acreditación estudiados, podemos ver que la Facultad de Agronomía realizó su proceso concibiendo a la misma como multidimensional y en el entendimiento de que es un proceso político. En ese sentido la Facultad se movió con los objetivos de mejora de la calidad, de fortalecimiento del vínculo universidad - sociedad y de la redefinición de la pertinencia. Al aplicar los mecanismos de participación se mostraron conscientes de que la calidad es la construcción de una negociación a partir de la comunidad en debate. De todas formas, se pudo constatar el riesgo de que se conciba a la comunidad exclusivamente como los actores internos a la institución, no incluyendo a la sociedad en esta construcción. En el marco de la visión política general, corresponde alertar sobre el hallazgo de la baja participación e implicancia de los estudiantes en estos procesos. De acuerdo a lo dicho, se previene sobre una desviación donde el proceso sea comandado por un núcleo de académicos comprometidos, corriendo el riesgo por un lado de reducir las visiones de la institución y por otro de caer en un proceso de características autoconfirmatorias e incluso de carácter tecnocrático.

Reconociendo los matices planteados, vemos que la Facultad de Agronomía se posicionó en este proceso promoviendo una acción democrática y participativa, en la búsqueda de fortalecer el valor de la mejora, y por tanto aportando a la definición del ARCUSUR hacia características de proceso emancipatorio (SAUL apud LEITE, 2005). 
El Sistema de Acreditación de Carreras de Grado del MERCOSUR (ARCU-SUR) en la Facultad de Agronomía de la Universidad de la República - Uruguay

En síntesis, en esta primera dimensión de conclusiones, la Universidad y la Facultad de Agronomía, marcaron una posición conceptual e ideológica clara, alejándose del enfoque tecnocrático y neoliberal, frente a estos procesos. Al momento de instrumentar la evaluación y la acreditación, esto se tradujo en metodologías e instrumentos coherentes con los conceptos, por lo que se puede considerar un aporte altamente positivo en la construcción del ARCUSUR, en especial la recreación práctica de una filosofía sobre la educación.

La dimensión instrumental. La Facultad de Agronomía se demostró una institución en continuo cambio y construcción y utilizó el proceso de autoevaluación como vehículo de reflexión y producción, lo que ineludiblemente la fortaleció. La búsqueda de la inclusión de los actores como profundización de la democracia, tuvo su expresión en los diversos instrumentos de participación de los mismos, que culminó con la formulación de un plan estratégico. La carrera de Agronomía cubrió todos los elementos para asegurar un productivo desarrollo de un proceso de autoevaluación, a saber: participación, compromiso, perspectiva amplia, visión académica e independencia (STUBRIN, 2005).

La estructura y presentación del informe de autoevaluación fue variando de un proceso a otro. Teniendo en cuenta que las características del mismo es ser técnico, representativo, analítico, equilibrado y realista, se concluye que los realizados por la carrera de Agronomía mostraron una gran heterogeneidad, donde el presentado al PEI se acercaba bastante a las características enunciadas (FACULTAD DE AGRONOMÍA, 2001a), el del MEXA no cumplía a cabalidad con estos requisitos, ya que respondía a las observaciones de los pares anteriores, y por último el realizado para el ARCU-SUR recuperó consistencia técnica. Asimismo el primero de estos informes, tenía lo que se entiende como desviación de excesivo criticismo, en tanto el del ARCU-SUR, si bien más equilibrado, se inclina más hacia la autoponderación. De todas formas, la institución realizó un verdadero aprendizaje, y pudo construir informes que produjeron conocimiento colectivamente acordado y con proyección de futuro.

En la instrumentación del proceso, se confirmó la premisa de que un proceso exitoso necesita de autoridades muy implicadas y con liderazgo, así como de un equipo técnico con conocimientos específicos en la gestión, y especialmente, que la coordinación se encuentre guiada por un organismo de carácter político. El principal déficit anotado es la disponibilidad de información fiable, lo que es un llamado de alerta para toda la Universidad para la construcción de instrumentos centrales. 
La dimensión de los efectos. Se observa que la carrera de Agronomía presenta un comportamiento sistemático, en tanto tomó iniciativas para responder a cada una de las sugerencias producidas por los informes de pares o por los dictámenes de acreditación.

Si bien se reconocieron resultados y efectos en todas las dimensiones, fue en la dimensión infraestructura y logística donde la institución identificó el mayor nivel de impacto. Esto caracterizó el proceso institucional transitado, en parte explicado porque las transformaciones académicas se venían procesando con anterioridad y presentaban escollos importantes, que el proceso de acreditación no logró vencer completamente. Cabe preguntarse si estos procesos funcionan exclusivamente en instituciones con cierto dinamismo, como la estudiada, o son capaces de producir rupturas fuertes o cambios de rumbo radicales en carreras que no presentan una cultura institucional de transformación.

Otro de los hallazgos constatados, es que los efectos se expanden más allá de la carrera en acreditación, como ejemplo se encuentra la diversificación de la oferta de carreras de grado y posgrado que se produjo en la Facultad.

Frente a todos los resultados producidos en Agronomía, y los hallazgos encontrados durante el estudio, se afirma que la institución ha cambiado. Entre el 2000 y el 2014 se produjeron una diversidad de acciones en las distintas dimensiones de la institución, derivadas o influenciadas por los procesos de acreditación, que han contribuido o jugado como motivadores o activadores de cambio en una dirección de mejora.

Finalmente, cabe reflexionar sobre la propia experiencia del ARCU-SUR, la que deberá ser estudiada desde diversas ópticas, a los efectos de complementar su evaluación. El SEM ha desarrollado una serie de proyectos y actividades que complementándose logran constituir una política integral con el potencial de generar un modelo regional autónomo, que sirva para la mejora y el fortalecimiento de la educación superior, así como herramienta de democracia y desarrollo de los países. Esta política se compone de tres grandes pilares, primero el sistema de acreditación, como promotor de la calidad de la actividad académica, potencialmente un generador de un espacio de desarrollo de conocimiento regional. El segundo, la movilidad de estudiantes y docentes a través de la construcción de redes diversas, en tanto fortalecimiento de la integración y desarrollo de la cultura y la cooperación regional. El tercero, el reconocimiento de títulos profesionales que promoverá la movilidad de profesionales, que se retroalimenta de los dos anteriores, y que promueve una integración del conocimiento y del desarrollo. 
El Sistema de Acreditación de Carreras de Grado del MERCOSUR (ARCU-SUR) en la Facultad de Agronomía de la Universidad de la República - Uruguay

\section{Referências}

ABADIE, Panambi. Una mirada desde la educación superior a la experiencia del Foro de Decanos de Agronomía del MERCOSUR. En Educación Superior y Sociedad. Nueva Época, Caracas, v. 14, n. 1, 2009.

BRASESCO, Gabriela. Reseña histórica de la Facultad de Agronomía. Trabajo realizado para el Curso de Epistemología 2007. Maestría en Ciencias Agrarias, Facultad de Agronomía, 2007. Disponible em:

<file:///C:/Users/usuario/Downloads/3_gabrielacruzbrasescohistoriafacultad(3).pdf>. Acceso em: 7 jun. 2014.

COMISIÓN AD HOC. Resolución de acreditación n01/04. Carrera de Agronomía de la Universidad de la República. Montevideo: Comisión Ad Hoc, 2004.

COMISIÓN AD HOC. Acuerdo de acreditación n²/09. Carrera de Agronomía de la Universidad dela República. Montevideo: Comisión AdHoc, 2009. Disponible em:

$<$ http://fagro2.fagro.edu.uy/index.php/institucional/evaluacion-institucionalacreditacion/acreditacion-2009>. Acceso en: 3 abr. 2015.

COMISIÓN AD HOC-pares externos. Informe final de acreditación de la carrera de Ingeniería Agronómica de laUdelaR. Montevideo: Comisión Ad Hoc, 2009. Disponible em: <http://fagro2.fagro.edu.uy/index.php/institucional/evaluacion-institucionalacreditacion/acreditacion-2009>. Acceso em: 5 mayo 2015.

DIAS SOBRINHO, José. Avaliação institucional, instrumento da qualidade educativa. Em: BALZAN, N.; DÍAS SOBRINHO, J. (Orgs.). Avaliação institucional: teoría e experiencias. San Pablo: Cortez Editora. 1995.

FACULTAD DE AGRONOMÍA. Anexo informe de autoevaluación institucional. Montevideo: Facultad de Agronomía - Universidad de la República, 2001a.

FACULTAD DE AGRONOMÍA. Informe de autoevaluación. Montevideo: Facultad de Agronomía - Universidad de la República, 2001b.

FACULTAD DE AGRONOMÍA. Informe de autoevaluación 2003. Montevideo:

Facultad de Agronomía - Universidad de la República, 2004.

FACULTAD DE AGRONOMÍA. Autoevaluación de la carrera de Ingeniero Agrónomo en el sistema ARCU-SUR. Montevideo: Facultad de Agronomía Universidad de la República. 2009a. Disponible en:

$<$ http://fagro2.fagro.edu.uy/index.php/institucional/evaluacion-institucionalacreditacion/acreditacion-2009>. Acceso en: 12 abr. 2015.

FACULTAD DE AGRONOMÍA. Formulario para la recolección de datos e información de la carrera de Ingeniería Agronómica. Montevideo: Facultad de Agronomía - Universidad de la República, 2009b. Disponible en:

$<$ http://fagro2.fagro.edu.uy/index.php/institucional/evaluacion-institucionalacreditacion/acreditacion-2009>. Acceso em: 22 feb. 2015

LANDINELLI, Jorge. La acreditación universitaria en el MERCOSUR: elementos para un balance experimental. En: COMISIÓN SECTORIAL DEL MERCOSUR - UdelaR.

MERCOSUR Educativo: el proceso de acreditación universitaria. Montevideo: UdelaR. 2007. p. 23-33.

LEITE, Denise. Reformas universitarias. Avaliação institucional participativa.

Petrópolis: Vozes, 2005. 
MARISQUIRENA, Gustavo. Ponencia. En Comisión Ad Hoc para ARCU-SUR (Org.). Taller nacional de evaluación ARCU-SUR. Montevideo: Comisión Ad Hoc para ARCU- SUR, 2011. Mimeo.

RME. Memorándum de entendimiento sobre la creación e implementación de un sistema de acreditación de carreras universitarias para el reconocimiento regional de la calidad académica de las respectivas titulaciones en el MERCOSUR y estados asociados. 2008. Disponible en

$<$ http://educacion.mec.gub.uy/mercosur/arcusur/arcu\%20sur\%20firmado.pdf $>$. Acceso en: 24 oct. 2014.

SANTOS, Boaventura Sousa. La universidad en el siglo XXI. Para una reforma democrática y emancipatoria de la universidad. Montevideo: Extensión universitaria, Universidad de la República - Trilce, 2010.

STUBRIN, Adolfo. Papel de la Evaluación y la Acreditación en la Relación entre la Universidad y el Estado. SEMINARIO LAS NUEVAS TENDENCIAS DE LA EVALUACIÓN Y LA ACREDITACIÓN EN AMÉRICA LATINA Y EL CARIBE. BUENOS AIRES, 2005.

UNESCO. Conferencia Mundial de Educación Superior. La educación superior en el siglo XXI: visión y acción. París: UNESCO. 1998.

UNIVERSIDAD DE LA REPÚBLICA. Estadísticas básicas de la UdelaR. Dirección de General de Planeamiento. Disponible en:

http://www.universidad.edu.uy/renderPage/index/pageId/452\#heading_1282>. Acceso en: 3 ene. 2015. 